\title{
Boundary Integral Equations - A Personal View
}

\author{
T. A. Cruse \\ Pagosa Springs, CO 81147
}

\section{Early Times}

My first exposure to what were best known then as potential methods was around the 1965-1966 academic year at the University of Washington in Seattle, Washington. I had been a stress analyst at The Boeing Company, working on the US's version of the Supersonic Transport, which was properly fated for many reasons never to be built and fly. As a stress analyst, I made extensive use of a mechanical calculator. I shared it with two other stress analysts, along with my very important stress books led, of course, by Roark's famous volume of stress results for beams, plates, shells, and the like.

I watched in envy as other engineers in the methods area gathered their computer output and punched cards each morning to continue the very first development of automated stress analysis methods for aircraft design (the Boeing 737 aircraft was to be that first design done by the finite element method not too long after this time). There were two types of computer algorithms that were still competing for this first design task - the force method and the displacement method. The force method derived from efficient hand analysis methods developed by Civil Engineers for analyzing complex redundant structures.

The displacement method was different. Not only did it involve an order of magnitude less computer input and output, it represented a paradigm shift to matrix methods and symbolics that greatly simplified all future computational mechanics. The computer-aided engineering revolution had begun, and I was privileged to observe those beginnings.

I decided that I wanted to be one of those engineers in the "methods group" at Boeing who were developing computer methods of structural analysis and found that I needed a Ph.D. degree to get in. I took a leave of absence from Boeing and enrolled in the Engineering Mechanics program at the University of Washington. One of the first faculty members I met was Frank Rizzo who had just completed his doctoral studies at the University of Illinois in Urbana and had come to the University of Washington as an Assistant Professor in the Civil Engineering Department.

When I met Professor Rizzo he was working with a graduate student who could program in Fortran. Frank's original dissertation was the formulation of the twodimensional elasticity problem using Betti's reciprocal work theorem. The Quarterly of Applied Mathematics rejected the manuscript derived from Frank's dissertation due to the absence of numerical results! So, the pressure was on to show that the elasticity problem could somehow be turned into an algorithm that worked at the same time that Professor Jaswon's efforts to get good numerical results for the biharmonic problem were failing. 
I had the distinct personal privilege to have been in the outer office when Frank came bounding in to declare, "It works!" And, indeed, he had directed the right algorithm to be programmed such that good numerical results were achieved. The Quarterly of Applied Mathematics did publish Frank's research results soon afterwards [i]. Several years ago I had the pleasure to successfully nominate Professor Rizzo for the ASME Worcester Reed Medal, which was presented to him based on that seminal paper.

What did he do that was so special?

While I certainly did not fully appreciate the critical issues of analytical integration of the "singular" terms, Frank seemed to understand its critical role. Or, the intrinsic simplicity of the analytical result appealed to him. Or, both. It is a lesson that is still important to those who work in this field. Always seek to use analysis rather than brute force numerics when you can. That is an issue that is of special importance in the more current use of isoparametric representations of the boundary geometry instead of the simple straight-line elements we used in the beginning.

What attracted me to this method? I found a number of factors very appealing. First, the potential methods (as they were named or referred to in those days) elegantly combined analytical results with computational algorithms. Second, everyone at the University was already working on new finite elements and I wanted something different. Finally, there was the enthusiasm and dedication of a really wonderful teacher in Frank Rizzo. This was a combination I could not resist.

I was enrolled after this time in an elastic wave propagation course taught by Professor Rizzo. As one of his graduate students I was searching for a suitable piece of original research upon which to base my dissertation. One day, Frank showed us how the Laplace transform converted the hyperbolic wave equation into an elliptic equation - I found my research topic at that point. I knew by then that the potential methods needed an elliptic governing equation to start with. I said that I would tackle the problem of wave propagation with the potential method and somehow address the inverse Laplace transform with its own numerical algorithm.

While I was never fully satisfied with the inverse transform problem, my research was successful. Waves did appear at the right place at the right time. One of the faculty on my dissertation committee even asked in disbelief if I had somehow programmed in the answer. I incorrectly thought that he was accusing me of cheating! Anyhow, he wasn't and I did receive my Ph.D. and published the results of my study [ii, iii]. Shortly thereafter I left for Carnegie-Mellon University and another change in direction.

\section{Elastic Fracture Mechanics}

I have had the pleasure of knowing some really good mechanics people as friends and colleagues over the years. The first was Frank Rizzo, who left Washington the year before I did (the year I did the actual dissertation) for the University of Kentucky. The second was Professor J. L. "Jerry" Swedlow at Carnegie-Mellon University. 
Jerry had recently joined CMU after a successful Ph.D. and post-Doc program at the California Institute of Technology. He had studied at CalTech under Professor Max Williams, who made some of the early critical contributions to fracture mechanics. Jerry had written one of the first successful elastoplastic finite element programs and was actively applying his work to model the critical role of the plastic zone ahead of the crack tip in two dimensions. Fracture mechanics was about in the same infancy as the finite element method.

Jerry recruited me to CMU in 1967 . He wanted someone to work on fracture mechanics with him, and we became close friends and colleagues until his untimely death over a decade ago. When I got to CMU, Jerry asked me if this potential method could be used to solve the problem of three dimension elastic cracks. No one had done any 3D work on cracks at that point.

I said "I don't know but I'll give it a go."

I then set about to formulate the equations for the three dimensional problem. I still have the thick notebook containing all of my analytical results from that and subsequent efforts. The notebook pages have colored corrections and dates from the many times I had to find errors in my analysis and correct them. Careful debugging of the computer codes was the only way I could verify my results, along with multiple derivations to get the same "correct" results. There were no algebraic programs in those days to work with.

The first paper posed an interesting challenge. What is a good 3D test problem that you can model using flat triangular elements? Certainly, modeling a cube meant that there were no geometry errors. However, only trivial test problems could be run for the uniform stress cases as the displacements and traction models were piecewise constants! And these did not satisfy a patch test. So, I compromised and hoped the reviewer would accept my hand-waving discussion of how the cube with one end fully-fixed somehow made sense when considering the case of a fixed-end cylinder problem. The shear stress is discontinuous at the corner between the fixed and free surfaces resulting in a stress singularity and the numerical results indicated this singularity.

The reviewer was kind and the first 3D potential method numerical solutions were published [iv]. However, these were not the crack problem I set out to solve. As I began to work on the crack problem, I got a telephone call in 1968 from the Westinghouse Research Center manager for computational mechanics, Dr. Steve Tuba. Steve said that they too were working on integral equation methods in three dimensions. My heart sank as I contemplated such competition early in my career. However, he then told me that they were having great numerical difficulties and asked if I would meet with them and explain why I was not having the same problems.

Very briefly, what I found was that they were programming the method of Kupradze [v]. There was some other literature at the time dealing with the superposition of single- and double-layer potentials for various problems [vi]. In all of these cases, the geometry was smooth at least in the sense of Liapunov (weak continuity of the normal). The 3D cube problem had edges and corners. In fact, as we now know, edges cause the unknown density function in this form of the potential method to have logarithmic singularities at each discontinuity of the normal. In the work at Westinghouse, the models 
had to have very large numbers of small elements near the edges and even then the results were not satisfactory.

I therefore coined two names for these methods to differentiate between the analytical approaches. The older, classical formulations of Kupradze and the Russian school I called the "indirect potential method." Indirect was used because the densities were not related to any physical boundary conditions and because these non-physical boundary densities had to be integrated to arrive at physical solution results. I named the method of Jaswon/Rizzo and myself, the "direct potential method" for the obvious reason that the unknown boundary data was the physical boundary conditions of the real problem. Often, it was only the boundary solution that was needed and we got this directly.

This terminology was first defined in the elastoplastic formulation paper [vii] and continued in the paper on 3D fracture solutions that I co-authored with my colleague from Westinghouse [viii]. However, the editor of the IJSS, Professor George Hermann, objected to using such a non-descriptive title as the "direct potential method." So, I coined the title boundary-integral equation (BIE) method.

Later, others would give the method the name boundary elements (both Professors Brebbia and Banerjee can rightly claim to have been involved in that renaming). I still call the formulation the BIE, and the numerical algorithms I call BEM.

That paper included an Appendix entitled Boundary Identity for the Stress Tensor, which turns out to have been a significant finding in itself. It was the first limit to the surface of the hypersingular Somigliana stress identity and was obtained after a proper, local regularization of the identity.

The paper also pointed out for the first time the degeneration that takes place in formulating the fracture mechanics problem in which two physical surfaces ideally occupy the same mathematical plane. More BIE insight on the modeling problem for cracks was given in the first national symposium dealing with surface cracks, organized by Professor Swedlow [ix]. Recently, both of these references were reprinted in a Milestone Paper series [x].

At this time, researchers in coalmine safety asked me whether or not the BIE method was competitive with finite elements for stress analysis. This was the first of what would be years of debate over this issue. I came to an early and easy solution. Sometimes the answer is "yes" and sometimes the answer is "no." In fact, I stated in 1974 that most of the time the answer was "no" but that the "yes" answer would apply to some classes of important problems - those with concentrated stresses including the fracture mechanics problems.

I was right then and believe that this is still the case. What we now call BIE or BEM is a very special and critically needed solution method, but its most effective applicability is for a small class of problems. Most problems that lack strong gradients of stress or strain are better solved by the finite element method. Such problems involve non-fracture driven plasticity, materials with significant inhomogeneities, plates and shells, etc. 
The use of piecewise constant boundary elements was not a satisfying formulation. I next formulated and implemented the linear case for 3D elasticity and applied this much more accurate code to a number of interesting problem sets [xi]. That paper introduced the necessary use of an indirect means for computing the coefficient of the jump term through the rigid body solution method. While accuracy increased and the algorithm satisfied a patch test, the degeneracy problem for fracture mechanics was still there.

\section{The Industrialization of BIE}

This is the time period when BEM and FEM really began to mature into design methods. Professor Carlos Brebbia invited me to Southampton University to consult on Jean-Claude Lachat's dissertation committee. The meeting was held as part of the first international meeting organized by Professor Brebbia [xii]. It was also the time that Professor Brebbia moved from his work in the FEM arena to the BIE arena. That effort would, in time, grow under Dr. Brebbia's leadership to become the Wessex Instititute. The experience introduced me to the importance and profound enjoyment of international collaborations.

Jean-Claude Lachat and Dr. Brebbia envisioned a BIE formulation that used the advanced algorithms being used in the FEM community based on isoparametric modeling for 3D surfaces. The modeling algorithms were modified by Jean-Claude Lachat for the BIE method and programmed by Professor John Watson [xiii].

I joined Pratt \& Whitney to expand my technical horizons in 1973. The first major professional meetings on the BIE method began following this move. The first was the meeting I organized with Professor Rizzo under the auspices of the ASME. The proceedings of that meeting included some important papers and ideas [xiv].

The second international BIE meeting was the (first) Symposium on Innovative Methods in Engineering, held in Versailles, France. Jean-Claude Lachat and I organized this meeting of international scholars and deliberately sought non-FEM papers. The meeting was a great professional and personal success as many new friendships were formed by all of us who attended this meeting.

This time period saw an enormous growth in interest in the BIE modeling approach that can be appreciated in the literature I reviewed in 1987 [xv]. My own work during this period focused largely on the fracture mechanics problem as applied to surface cracks [xvi, xvii] I recently had the opportunity to review 25 years of fracture mechanics work using the BIE method [xviii].

The BIE-degeneration for fracture mechanics problems continued to occupy my thoughts over a number of years. The use of analytical Green's functions is limited to the 2D problem [xix]. Work that I began, but did not publish, while at Pratt \& Whitney, was further developed after I went to Southwest Research Institute in 1983. This work led to the formulation of the traction-BIE for cracks that models a single surface of discontinuity using the hypersingular Somigliana identity [xx].

During that period until now the literature on hypersingular equations has been expanding at a very large rate. Issues have been identified and debated at length 
regarding the extremes from the Hadamard finite part approaches to my position on selfregular BIE formulations. The literature is too new and unsettled to draw any firm conclusions. But, I believe we are close to a proper formulation for the fracture mechanics problem. After all this time, we should have one!

\section{Acknowledgments}

Of all the many technical areas I have worked, the one that still interests me the most intellectually is the BIE area. It still challenges us to have good formulations and, even more, to have good computational algorithms. BIE was the first interest I had and it continues.

I believe that my continued involvement in BIE over the years as I made a number of career changes is partially due to the friendships and interactions with the quality individuals in this community during that time. In addition to Professors Rizzo and Brebbia, I want to acknowledge Professor Banerjee with whom I worked very closely when I was at Pratt \& Whitney, along with Dr. Ray Wilson of PWA. From my years in leadership of the International Association of Boundary Element Methods, I have the great pleasure to work closely with such great colleagues as Professors Luigi Morino, Wolfgang Wendland, Shoichi Kobayashi, Masataka Tanaka, Subrata Mukherjee, Giulio Maier, Satya Atluri and so many others. Thanks to all these fine people, this is still a highly active and stimulating area of research.

i. F. J. Rizzo, An integral equation approach to boundary value problems of classical elastostatics, Quarterly of Applied Mathematics, 25, 83-95, 1967.

ii. T. A. Cruse and F. J. Rizzo, A direct formulation and numerical solution of the general transient elastodynamic problem, I, Journal of Mathematical Analysis and Applications, 22, 1, 244-259, 1968.

iii. T. A. Cruse, A direct formulation and numerical solution of the general transient elastodynamic problem, II, Journal of Mathematical Analysis and Applications, 22, 2, 341-355, 1968.

iv. T. A. Cruse, Numerical solutions in three dimensional elastostatics, International Journal of Solids \& Structures, 5, 1259-1274, 1969.

v. V. D. Kupradze, Potential methods in the theory of elasticity, Davey, New York, 1965.

vi. C. E. Massonet, Numerical use of integral procedures, Stress Analysis, edited by O. C. Zienkiewicz and G. S. Hollister, J. Wiley, 1965.

vii. J. L. Swedlow and T. A. Cruse, Formulation of boundary integral equations for three-dimensional elasto-plastic flow, International Journal of Solids \& Structures, 7, 1673-1683, 1971.

viii. T. A. Cruse and W. VanBuren, Three-dimensional elastic stress analysis of a fracture specimen with an edge crack, International Journal of Fracture Mechanics, 7, 1, 1-15, 1971. 
ix. T. A. Cruse, Numerical evaluation of elastic stress intensity factors by the boundary-integral equation method, in The Surface Crack: Physical Problems and Computational Solutions, Edited by J. L. Swedlow, ASME, New York, 153-170, 1972.

x. R. J. Sanford, Editor, Selected papers on Crack Tip Stress Fields, SEM Classic Papers Volume CP 2, SPIE Milestone Series, Volume MS 138, 1997.

xi. T. A. Cruse, An improved boundary-integral equation method for threedimensional elastic stress analysis, Computers \& Structures, 4, 741-754, 1974.

xii. T. A. Cruse, Application of the boundary-integral equation solution method in solid mechanics, Opening Lecture in the International Conference on Variational methods in Engineering, Southampton University, England, September 25-29, 1972.

xiii. J. C. Lachat and J. O. Watson, Effective numerical treatment of boundaryintegral equations, International Journal of Numerical Methods in Engineering, 10, 991-1005, 1974.

xiv. T. A. Cruse and F. J. Rizzo (Eds.), Boundary-integral equation method: computational applications in applied mechanics, ASME AMD-Vol. 11, New York, 1975.

xv. T. A. Cruse, Recent advances in boundary element analysis methods, Computer Methods in Applied Mechanics and Engineering, 62, 227-244, 1987.

xvi. T. A. Cruse and P. M. Besuner, Residual life prediction for surface cracks in complex structural details, Journal of Aircraft, 12, 4, 369-375, 1975.

xvii. T. A. Cruse and G. J. Meyers, Three-dimensional fracture mechanics analysis, Journal of the Structural Division, 103, ST2, ASCE, 309-320, 1977.

xviii. T. A. Cruse, BIE fracture mechanics: 25 years of developments, Computational Mechanics, 18, 1-11, 1996.

xix. M. D. Snyder and T. A. Cruse, Boundary integral equation analysis of cracked anisotropic plates, International Journal of Fracture, 11, 2, 315-328, 1975.

xx. E. Z. Polch, T. A. Cruse, and C.-J. Huang, Traction-BIE solutions for flat cracks, Computational Mechanics, 2, 253-267, 1987. 